\title{
Editorial: Special issue on social human-robot interaction for human-care robots
}

\author{
Minsu Jang ${ }^{1} \cdot$ Ho Seok Ahn ${ }^{2} \cdot$ Jong-Suk Choi ${ }^{3} \cdot$ Franziska Kirstein $^{4} \cdot$ Luis Yoichi $^{5}$
}

Published online: 5 July 2021

(c) The Author(s), under exclusive licence to Springer-Verlag GmbH Germany, part of Springer Nature 2021

Social human-robot interaction aims to replicate human intelligence for interpersonal communication so that robots can communicate with human users in a more natural and safe way-a human way. It is well perceived that social interaction significantly promotes efficacy of personal robot services in various areas, e.g., telepresence, elderly care, rehabilitation, education, intervention therapy and guidance [1-4]. Moreover, recent studies increasingly have provided interesting clues that social interaction improves productivity, promotes safety and reduces users' psychological tension while working with professional service robots, e.g., industrial collaborative robots, delivery robots and multior-swarm robots [5, 6]. It is our mission with this special issue to introduce recent work done in designing, implementing and validating technical approaches to realize social human-robot interaction.

This special issue on social human-robot interaction for human-care robots presents five papers that were selected by the guest editors, Minsu Jang, Ho Seok Ahn, Jong-Suk Choi, Franziska Kirstein and Luis Yoichi, based mainly on their relevance and contributions to human-robot interaction. The

Minsu Jang

minsu@etri.re.kr

Jong-Suk Choi

cjs@kist.re.kr

Franziska Kirstein

fk@blue-ocean-robotics.com

Luis Yoichi

yoichims@ieee.org

1 Electronics and Telecommunications Research Institute, Daejeon, South Korea

2 University of Auckland, Auckland, New Zealand

3 Korea Institute of Science and Technology, Seoul, South Korea

4 Blue Ocean Robotics, Odense, Denmark

5 Nagoya University, Nagoya, Japan papers went through the normal multistage peer review process of the ISR journal.

The papers in this special issue investigate three different but equivalently important aspects of human-robot interactions: (1) robust interactions in the real world, (2) strategic linguistic verbal interactions and (3) evaluation of the robotic service quality.

Robustness of perception and control is essential for a robot to be accepted as a trustworthy companion by human users, as it allows robots to avoid out-of-context behaviors or responses in the real world. In "Investigating the importance of shape features, color constancy, color spaces, and similarity measures in open-ended 3D object recognition," authors propose an approach of effectively combining color and shape features to improve the performance of 3D object recognition in the real world. It is noticeable that their method is data efficient in learning new objects, which helps robots efficiently adapt to environment changes and perform interactions with improved robustness. In "Gait pattern generation algorithm for lower-extremity rehabilitation-exoskeleton robot considering wearer's condition," authors introduce a method for generating gait patterns according to the wearer's intention and environment conditions and present experimental results that show their method could successfully create adaptive strides that are better suited for the user. The paper showcases that adaptiveness is an essential element in control for robust physical interactions between humans and robots.

Linguistic verbal interaction is the most common form in social human-robot interaction. Little is studied on how a robot should initiate and maintain dialogs with human users for increased engagement, sustainable interactions and improved task performance. In "Changes in linguistic behaviors based on smart speaker task performance and pragmatic skills in multiple turn-taking interactions" and "Towards a Conversational Model for Counsel Robots: How Different Question Types Elicit Different Linguistic Behaviors," authors present experimental results and analyses on which 
elements of robot interaction skills affect user's linguistic behaviors and how a robot can strategically maintain conversations with human users to be a better robotic counselor. These studies are preliminary but are very important because we believe that the topics these papers deal with shed light on the way to intelligent, natural and open linguistic verbal interactions with robots.

Assessing the quality of robot services is a very difficult task as it is not possible in most cases to depict quantitative measures. In "Case studies on the usability, acceptability and functionality of autonomous mobile delivery robots in real-world healthcare settings," authors present qualitative assessment of robot delivery services for health care through participatory surveys and analysis. The robot used in the experiment is not equipped with features for human-robot interaction, but it provides inspirations on how the quality of social interactions could be measured in the respect of usability, acceptability and functionality.

Finally, we would like to express our great appreciation to the authors for their enthusiastic cooperation in all the steps of reviews and revisions to make this special issue. We hope that the readers enjoy this special issue.

\section{References}

1. Broekens J, Heerink M, Rosendal H (2009) Assistive social robots in elderly care: a review. Gerontechnology 8(2):94-103

2. Anzalone SM, Boucenna S, Ivaldi S, Chetouani M (2015) Evaluating the engagement with social robots. Int J Soc Robot 7(4):465-478

3. Belpaeme T, Kennedy J, Ramachandran A, Scassellati B, Tanaka F (2018) Social robots for education: a review. Sci Robot 3(21):eaat5954

4. Mubin O, Ahmad MI, Kaur S, Shi W, Khan A (2018) Social robots in public spaces: a meta-review. In: International conference on social robotics, pp 213-220. Springer, Cham

5. Fischer K (2019) Why collaborative robots must be social (and even emotional) actors. Techné: Res Philos Technol 23(3):270-289

6. Görür OC, Rosman B, Sivrikaya F, Albayrak S (2018) Social cobots: anticipatory decision-making for collaborative robots incorporating unexpected human behaviors. In: Proceedings of the 2018 ACM/IEEE international conference on human-robot interaction, pp 398-406

Publisher's Note Springer Nature remains neutral with regard to jurisdictional claims in published maps and institutional affiliations. 